This item was submitted to Loughborough's Research Repository by the author.

Items in Figshare are protected by copyright, with all rights reserved, unless otherwise indicated.

\title{
Close coupled resonant aperture inserts for waveguide filtering applications
}

PLEASE CITE THE PUBLISHED VERSION

http://dx.doi.org/10.1109/7260.915620

\section{PUBLISHER}

(C) Institute of Electrical and Electronics Engineers (IEEE)

\section{VERSION}

VoR (Version of Record)

\section{LICENCE}

CC BY-NC-ND 4.0

\section{REPOSITORY RECORD}

Seager, R.D., J.C. Vardaxoglou, and David S. Lockyer. 2019. "Close Coupled Resonant Aperture Inserts for Waveguide Filtering Applications". figshare. https://hdl.handle.net/2134/9443. 
This item was submitted to Loughborough's Institutional Repository (https://dspace.lboro.ac.uk/) by the author and is made available under the following Creative Commons Licence conditions.

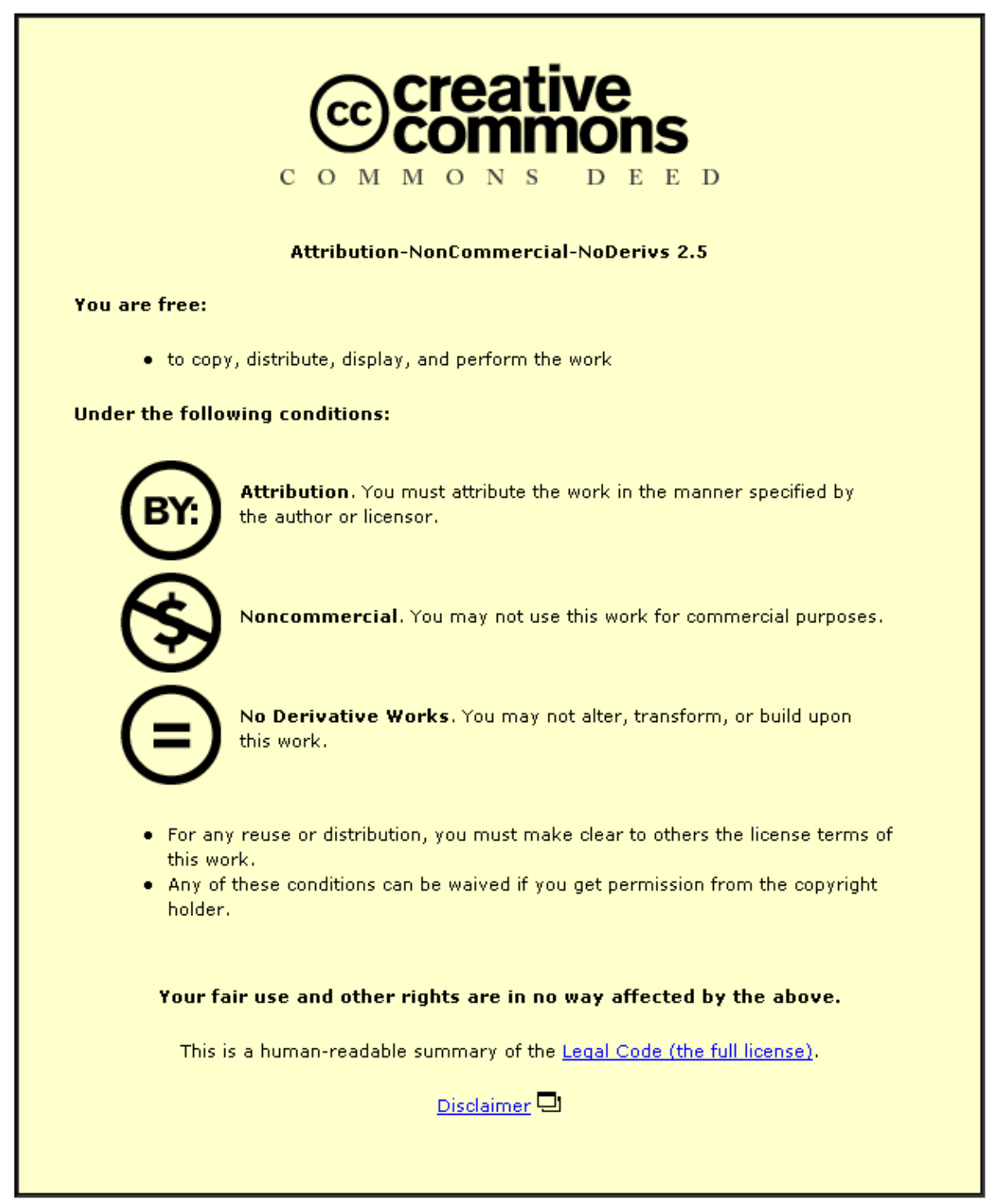

For the full text of this licence, please go to: http://creativecommons.org/licenses/by-nc-nd/2.5/ 


\title{
Close Coupled Resonant Aperture Inserts for Waveguide Filtering Applications
}

\author{
R. D. Seager, Member, IEEE, John C. Vardaxoglou, Member, IEEE, and D. S. Lockyer
}

\begin{abstract}
A two-layer frequency selective surface (FSS) is used as a very compact and lightweight transverse waveguide filter element. A narrow-band transmission response is produced from two layers, which, otherwise, in isolation would exhibit a broadband response. Measured results are compared with theoretical data obtained from a Transmission Line Matrix (TLM) based modeling method. Reduction in the passband bandwidth of a factor of four has been achieved using the proposed structure.
\end{abstract}

Index Terms-Frequency selective surfaces, TLM, waveguide filters.

\section{INTRODUCTION}

$\mathbf{T}$ RADITIONALLY, to produce filtering responses in waveguides, cavities or reactive components such as irises and posts, in conjunction with $\mathrm{J}$ and $\mathrm{K}$ inverters, are used [1]. Both the cavities and inverter sections are bulky in waveguides and waveguide filters tend to be relatively large and heavy. In contrast, by using a resonant structure as a transverse insert, one can achieve good filtering performance but with a much more compact and lightweight design.

In FSS technology, an array of resonant apertures within a metallic sheet yields a bandpass transmission response. If a section of such an array is inserted transversely in a rectangular waveguide, the array response can be maintained if the inner dimensions of the waveguide contain an integer number of the standard FSS array's unit cells [2], [3]. Further, the FSS insert will occupy less than $200 \mu \mathrm{m}$ of waveguide length. When considering thin slotted rectangular apertures the slot length primarily determines the resonant frequency, in that it is approximately a $\lambda / 2$ resonator. In a waveguide close to cutoff, the slot length can be accommodated across the width of the waveguide but the response is broadband and therefore is of limited use in filtering applications. However, it has been previously demonstrated that the use of a two-layer aperture FSS, under plane wave illumination, can significantly reduce the bandwidth of the transmission response [4]. This letter presents results on the application of close coupled aperture frequency selective surfaces (FSS) specifically for waveguide filtering applications. We investigate the potential of this structure to act as a bandpass filtering element rather than as a filter in its own right. This involves using two separate aperture arrays either side of a single dielectric layer, and if a relative shift is introduced between the

Manuscript received July 26, 2000; revised December 6, 2000.

The authors are with the Department of Electronic and Electrical Engineering, Loughborough University, Loughborough Leicestershire, LE11 3TU, U.K. (e-mail: r.d.seager@lboro.ac.uk; j.c.vardaxoglu@lboro.ac.uk; d.s.lockyer@lboro.ac.uk)

Publisher Item Identifier S 1531-1309(01)03208-1.
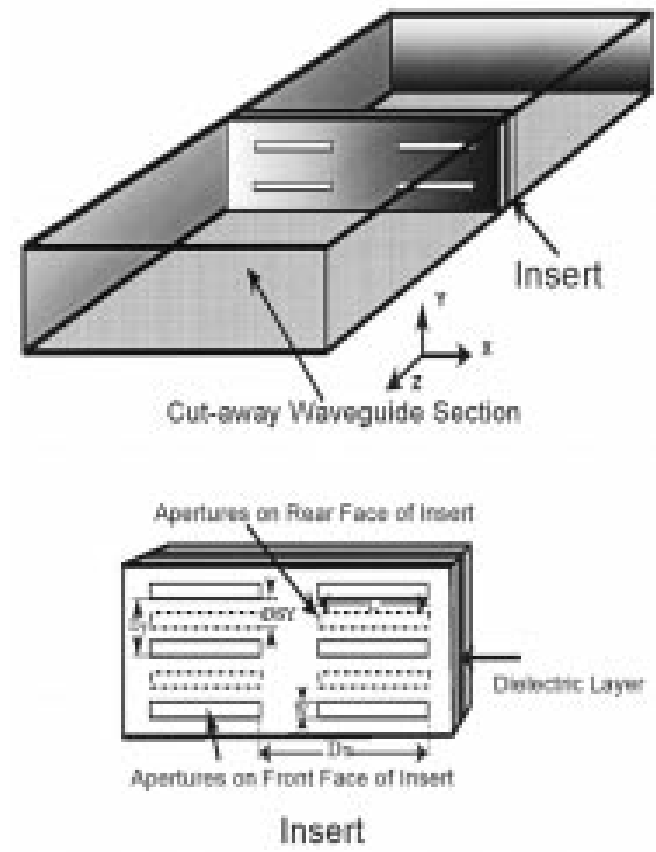

Fig. 1. Schematic of evaluated system.

layers strong coupling fields are established in the intermediate dielectric region. In this situation there is a narrowing of the transmission response bandwidth and the slotted elements behave as though they are longer electrically than physically.

A schematic representation of the structure under investigation is shown in Fig. 1, where a double layer aperture FSS is transversely inserted inside a WG-16 $(22.86 \mathrm{~mm} \times 10.16 \mathrm{~mm})$ waveguide used in this study [5]. The structure of the insert is also shown in Fig. 1. It consists of two copper sheets containing a finite array of slotted apertures (length $8.3 \mathrm{~mm}$ and width 0.3 $\mathrm{mm})$, which are separated and supported by a thin dielectric (polyester) layer of thickness $75 \mu \mathrm{m}$ and $\varepsilon_{r}=3$. The periodicities in $x$ and $y$ are $D_{x}=11.5 \mathrm{~mm}$ and $D_{y}=3.39 \mathrm{~mm}$ respectively, which means that the waveguide accommodates two $x$ periods and three $y$ periods. We also introduce the relative shift between layers in the $y$ direction, $D S Y$, which in this case is $D y / 2=1.69 \mathrm{~mm}$. It is the introduction of this shift that is necessary to induce the high coupling fields between arrays such that the required transmission response can be obtained.

\section{TRANSMISSION LINE MATRIX (TLM) MODEL}

TLM is a time domain method [6], [7] and as such yields the fields within the defined workspace cells as well as results over 
a given frequency range for one simulation. The fields can give increased insight into the functioning of the component being modeled. A very powerful reason for using TLM is the fact that it can model dielectric and metallic losses as well as thin metal layers accurately, and therefore gives a model that approximates the measured structure well.

The $\mathrm{TE}_{10}$ mode is launched in the waveguide using a presimulation to calculate the transverse field structure at cutoff, with the waveguide ends matched to minimize reflections in the model. The transverse inserts are placed halfway down the waveguide and four output points, two before and two after the insert, are used to sample the time domain signal. Each pair of points defines a port used to extract the scattering parameters, with the port positions taken to be in the middle of the output point pairs. By considering the separation of the pairs of output points and the fields at them, it is possible to obtain the forward and reverse going waves at the input and output ports, allowing calculation of the scattering parameters.

The software used here [8] allows for inclusion of both dielectric and metallic losses. However, neither of these parameters are frequency dependant in this implementation of TLM and values are selected to be accurate at the center frequency of the designed insert response. The complete model consists of 257000 cells and runs for about 30 Hours CPU time (on a Sun Ultra Enterprise 3500 server, using two $336 \mathrm{MHz}$ processors and 2 GB of memory) before a stable solution is reached. The cell dimensions and dielectric constant define the upper frequency of the model in that at least ten cells per wavelength are recommended for accurate results. Data is present in the solution for frequencies above this maximum value, so the time domain data is filtered and resampled to avoid aliasing when the Fourier Transform is performed.

\section{Measured AND Simulated Results}

Fig. 2 shows measured and simulated results for a single slot of length $16.5 \mathrm{~mm}$ and simulated results for a single slot of 8.3 $\mathrm{mm}$ and a double layer insert with slot lengths of $8.3 \mathrm{~mm}$ and $D S Y=0$. This shows that the $16.5 \mathrm{~mm}$ slot has a resonance very close to the cutoff frequency, which makes it unsuitable for filtering applications. The passband itself is centered around $7.5 \mathrm{GHz}$ and exhibits a $3 \mathrm{~dB}$ bandwidth of about $2.4 \mathrm{GHz}(32 \%)$. EVALUATED STRUCTUREThe discrepancy between the measured and simulated results for this slot is probably due to the dielectric having been removed from the slot. At frequencies near the cutoff the angle of incidence, $\theta$, of the $\mathrm{TE}_{10}$ mode is very oblique as given by $\theta=\sin ^{-1}\left(f_{c} / f\right)$, where $f_{c}$ is the cutoff frequency of the waveguide (in this case $6.557 \mathrm{GHz}$ ) and $f$ is the operating frequency $(\mathrm{GHz})$. In the case of the measurement, the edges of the slot where the dielectric had been removed would have had the dielectric on the back exposed to the propagating mode at these angles of incidence. The interference from the dielectric is likely to have an adverse effect on the measurement and could lead to the observed discrepancy. Fig. 2 also shows the simulated results for a double layer insert with slot lengths of $8.3(D S Y=0)$ and a single $8.3 \mathrm{~mm}$ slot. The simulation of the insert shows a broad resonance at about $15 \mathrm{GHz}$, which is well above the normal operational band of the waveguide. The

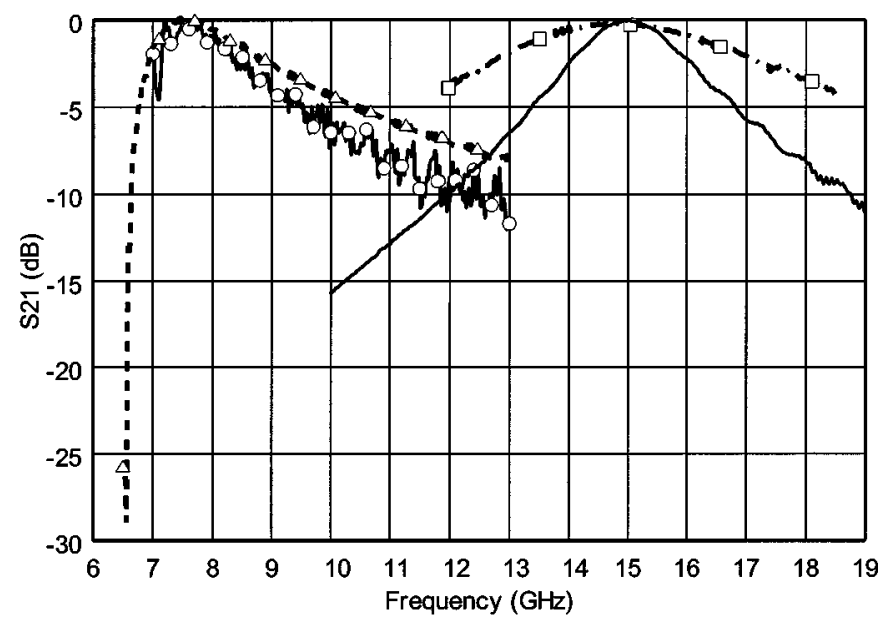

Fig. 2. Measured and modeled S21 data for single apertures. - - $\triangle$ - - Modeled $16.5 \mathrm{~mm}$ aperture. $\bigcirc-$ Measured $16.5 \mathrm{~mm}$ aperture. $-\square-$ - - Modeled result of two layer insert with zero offset for comparison. - Modeled $8.3 \mathrm{~mm}$ aperture.

bandwidth is about $5 \mathrm{GHz}$, which represents $33 \%$. The resonance of the single $8.3 \mathrm{~mm}$ slot is shown for reference. It is clear that whilst the resonance is well away from the cutoff, the bandwidth is large, which limits the filtering applications for which this device can be considered.

When an insert including the relative shift, $D S Y=1.69$ $\mathrm{mm}$, is introduced however, the performance improves considerably. A photograph of the insert is shown in Fig. 3(a). Fig. 3(b) shows the results for this insert with two different dielectric support layers (polyester and Kapton). The first point to note is that the measured and predicted results (for the polyester substrate) agree very well and describe a resonant frequency around 10 $\mathrm{GHz}$ together with an in band loss of about $7 \mathrm{~dB}$. The $3 \mathrm{~dB}$ bandwidth has decreased from $5 \mathrm{GHz}(D S Y=0)$ to about 800 $\mathrm{MHz}(8 \%)$ in this case, and this yields a good potential filter. The $7 \mathrm{~dB}$ loss observed in the measurement has been accurately reproduced using a value for $\tan \delta$ of 0.06 . This observed loss is exacerbated by the high coupling fields established in the separation region between the arrays. There is also likely to be a contribution from the adhesive that bonds the laminate together. The precise thickness and dielectric properties of this adhesive are unknown, but it is likely that in this sensitive structure, together with the high coupling fields, it contributes to the loss. Fig. 3(b) also shows the measured result of the same insert design on a lower loss Kapton dielectric ( $\tan \delta \approx 0.002)$. The loss in this case is reduced to around $3.5 \mathrm{~dB}$ whilst the bandwidth has been maintained at $800 \mathrm{MHz}$, and this demonstrates that if a very low loss material were used the passband loss would decrease still further. There is a small shift in the resonant frequency for this structure, to $10.6 \mathrm{GHz}$, that may be due to a slight decrease in the coupling between arrays due to the extra thickness of the Kapton (108 $\mu \mathrm{m}$ compared to $75 \mu \mathrm{m})$. For indication purposes the simulated case for the insert when $\tan \delta=0$ is also included in Fig. 3(b) (thereby leaving only metallic losses in the model) and shows that a lossless dielectric would yield very close to zero passband loss.

In order to verify that the fields in the dielectric play a key role in the production of these filtering elements, we have plotted the 


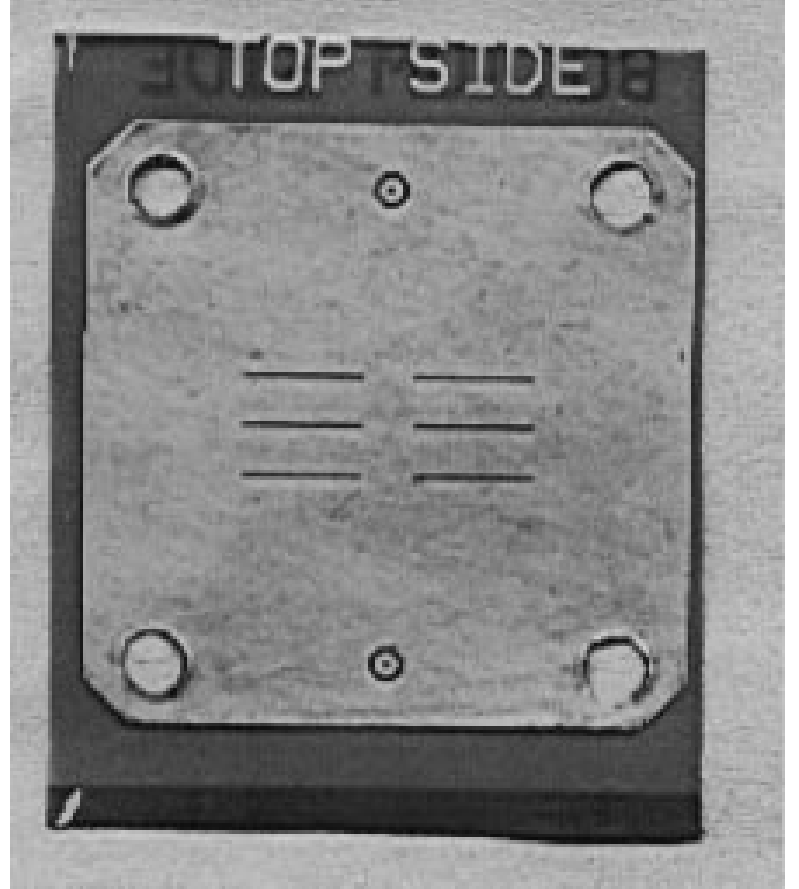

(a)

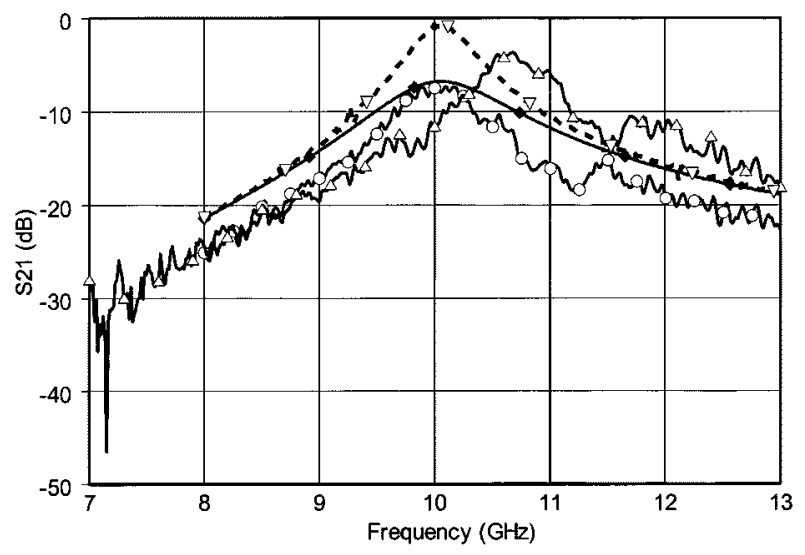

(b)

Fig. 3. (a) Photograph of two layer insert, (b) measured and modeled S21 data for the two layer insert. $-\bigcirc-$ Measured with polyester dielectric. $-\triangle$ Measured with kapton dielectric. $\longrightarrow$ Modeled with $\tan \delta=0.06--\nabla$ Modeled with $\tan \delta=0.0$

simulated electric field at $10 \mathrm{GHz}$ in the dielectric, and this is shown in Fig. 4. The dielectric is modeled as being three cells long in the $z$-direction, and this field (in the $x-y$ plane) representation is taken from the cell adjacent to the front array of the insert. The slots on the front and rear arrays are marked but can be easily identified from the magnitudes of the electric fields, where the area of the front slots (yellow/orange) has a higher field than that of the rear slots (blue). The important point to note is the very high field regions (red) in the areas that are bounded, front and rear, by metal. These are the high coupling fields established due to the shift $D S Y$, and are the result of the incident energy having to progress from the front slots to the rear slots through the thin separation region bounded by copper.

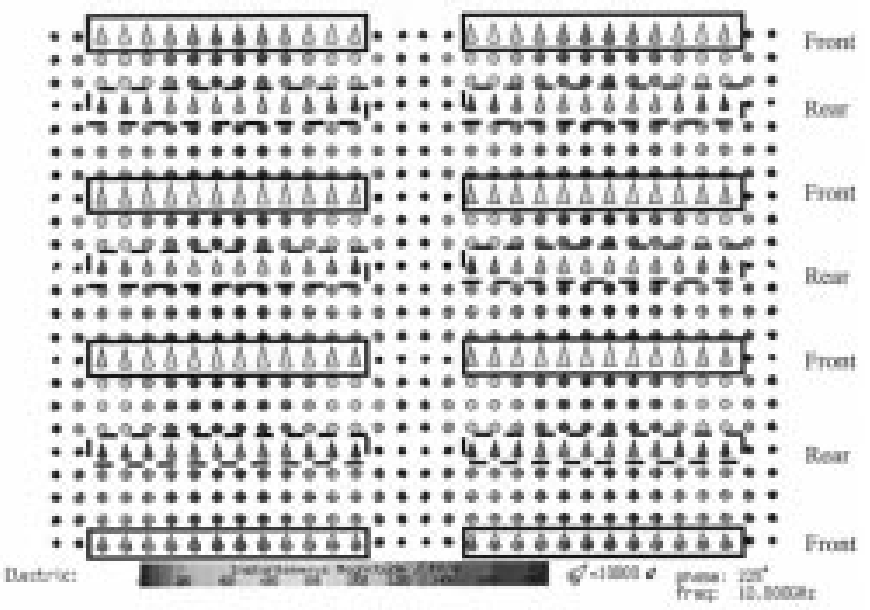

Fig. 4. Electric fields in dielectric between aperture arrays. $\square$ Aperture position on front of insert. [- . - $\mid$ Aperture position on rear of insert.

\section{CONCLUSIONS}

We have presented results demonstrating the potential to produce a bandpass type response in a waveguide using a twosided array of close-coupled resonant aperture FSS. The response has a much improved performance in terms of bandwidth over both that of a single layer operating at a similar frequency and the same insert without a relative shift between layers. In both cases this leads to a reduction in percentage bandwidth of a factor of four. The TLM model has been successfully used to model the structure accurately and has provided valuable information regarding the fields contained in the separation region. The propensity of the narrow-band two-layer structure to exhibit losses at resonance has been identified and attributed to dielectric losses. We have anticipated that a very low loss dielectric would virtually eliminate these losses. These waveguide filter elements represent a considerable potential saving in weight and size over conventional designs, with the example studied here being only a fraction of a millimeter thick.

\section{REFERENCES}

[1] R. E. Collin, Foundations for Microwave Engineering, 2nd ed. New York: McGraw Hill, 1992, ch. 8.

[2] W. Yu, S. Dey, and R. Mittra, "On the modeling of periodic structures using the finite-difference time-domain algorithm," Microwave Opt. Technol. Lett., vol. 24, no. 3, pp. 151-155, 2000.

[3] W. Yu, R. Mittra, and D. H. Werner, "FDTD modeling of an artificially synthesized absorbing medium," IEEE Microwave Guided Wave Lett., vol. 9, pp. 496-498, Dec. 1999.

[4] D. S Lockyer and J. C. Vardaxoglou, "Reconfigurable FSS from two layers of slotted dipole arrays," Electron. Lett., vol. 32, pp. 512-513, 1996.

[5] R. Pous and D. M. Pozar, "A frequency-selective surface using aperture coupled microstrip patches," IEEE Trans Antennas Propagat., vol. 39, pp. 1766-1769, Dec. 1991

[6] P. B. Johns and R. L. Beurle, "Numerical solutions of 2-dimensional scattering problems using a transmission line matrix," Proc. Inst Elec. Eng., vol. 119, no. 8, pp. 1203-1208, Sept. 1971.

[7] W. J. R. Hoeffer, "The transmission line matrix method-Theory and applications," IEEE Trans. Microwave Theory and Techniques, vol. MTT-33, no. 10, Oct. 1985.

[8] MicroStripes Version 4.0. Nottingham, U.K.: Kimberley Communications Consultants. 